\title{
Application of the screening and indirect cohort methods to evaluate the effectiveness of pneumococcal vaccination program in adults 75 years and older in Taiwan
}

Wei-Ju Su ${ }^{1,2}$, Pei-Hung Chuang ${ }^{3,4}$, Luan-Yin Chang ${ }^{2}$, Hsiu-Yun Lo ${ }^{1}$, Chuen-Sheue Chiang ${ }^{1}$, Ez-Tzu Wang ${ }^{1}$ and Chin-Hui Yang ${ }^{1 *}$ (D)

\begin{abstract}
Background: The Taiwanese national 23-valent pneumococcal polysaccharide vaccine (PPV23) program in adults $\geq 75$ years of age and the 13-valent pneumococcal conjugate vaccine (PCV13) program for children were implemented in 2008 and 2013, respectively. In this study we evaluated PPV23 vaccine effectiveness (PPV23VE) in the elderly, with regard to both direct protection from the vaccine itself and the indirect protection conferred by PCV13 immunization in children.

Methods: The incidence of invasive pneumococcal disease (IPD) in Taiwan from July 2008 to June 2016 was collected from IPD surveillance data. A comparison of IPD incidence with a nationwide vaccination registry allowed an estimation of PPV23VE by the screening and indirect cohort methods.

Results: The incidence of IPD in adults $\geq 75$ years of age ranged from 13.9 per 100,000 inhabitants during the period July 2008-June 2013 to 10.4 per 100,000 inhabitants between July 2013 and June 2016 (relative risk [RR]: 0.75; 95\% confidence interval [95\% Cl]: 0.67-0.85). According to the screening method, PPV23VE against death within 30 days of IPD onset, all IPD, and PPV23-serotype IPD was 32.5\% (95\% Cl: 17.5-44.7\%), 33.9\% (95\% Cl: 25.241.5\%) and 43.4\% (95\% Cl: 34.4-51.2\%), respectively. PPV23VE with the indirect cohort method was 39.0\% (95\% Cl: 15.5-55.9\%) for all PPV23 serotypes and 71.5\% (95\% Cl: 44.2-85.4\%) for 11 serotypes included in PPV23 but not in PCV13. During the period July 2008-June 2012, PPV23VE against PPV23-serotype IPD was $55.1 \%$ (95\% Cl: 27.272.3\%).
\end{abstract}

\footnotetext{
* Correspondence: inf@cdc.gov.tw; yangch55@gmail.com

'Division of Acute Infectious Diseases, Centers for Disease Control, Ministry of Health and Welfare, 6, Linsen South Road, Taipei 100, Taiwan, Republic of China

Full list of author information is available at the end of the article
}

(C) The Author(s). 2021 Open Access This article is licensed under a Creative Commons Attribution 4.0 International License, which permits use, sharing, adaptation, distribution and reproduction in any medium or format, as long as you give appropriate credit to the original author(s) and the source, provide a link to the Creative Commons licence, and indicate if changes were made. The images or other third party material in this article are included in the article's Creative Commons licence, unless indicated otherwise in a credit line to the material. If material is not included in the article's Creative Commons licence and your intended use is not permitted by statutory regulation or exceeds the permitted use, you will need to obtain permission directly from the copyright holder. To view a copy of this licence, visit http://creativecommons.org/licenses/by/4.0/ The Creative Commons Public Domain Dedication waiver (http://creativecommons.org/publicdomain/zero/1.0/) applies to the data made available in this article, unless otherwise stated in a credit line to the data. 
(Continued from previous page)

Conclusions: PPV23 is able to prevent IPD and 30-day fatality in adults 75 years of age and older due to a combination of direct effects from PPV23 and indirect effects from PCV13. It might confer higher protection against PPV23-serotype IPD before the introduction of PCV13 program in children.

Keywords: Pneumococcal polysaccharide vaccines, Vaccine effectiveness, Invasive pneumococcal disease, Screening method, Indirect cohort method

\section{Background}

Invasive pneumococcal disease (IPD), defined as the isolation of $S$. pneumoniae from a normally sterile body site, such as the blood, cerebrospinal fluid or pleural effusion, poses significant health threats to young children ( $<5$ years of age), older adults, and individuals with chronic medical conditions $[1,2]$. In the USA, a national pneumococcal conjugate vaccine $(\mathrm{PCV})$ program for children was introduced in 2000, which was earlier than most countries worldwide [3]. The pediatric population was chosen because the incidence of IPD was higher in children under the age of 5 years (71.8 per 100,000 inhabitants) than in adults $\geq 65$ years ( 57.6 per 100,000 inhabitants) [4]. However, by 2016, the main disease burden of IPD in the USA had shifted from children $<5$ years ( 8 per 100,000 inhabitants) to adults $\geq 65$ years $(24$ per 100,000 inhabitants) [5]. Similar findings were determined in Taiwan from the pre- to the post-PCV immunization era [3, 6]. Age-related changes in IPD epidemiology over time indicate the need to evaluate the impact of pneumococcal immunization programs and vaccine effectiveness (VE) both in children and in older adults $[3,7,8]$.

National 23-valent pneumococcal polysaccharide vaccine (PPV23) and 13-valent PCV (PCV13) vaccination programs were started in Taiwan in 2008 and 2013, respectively [3, 6, 9]. Thus, any evaluation of the protective effects of PPV23 in the elderly must take into account both direct protection from PPV23 vaccination and indirect protection from PCV13 immunization in children [10].

While evidence-based recommendations form the cornerstone of the vaccination policy of the World Health Organization (WHO) and of many developed countries, such data are scarce in Africa and Asia [7, 11, 12]. Available systematic reviews and meta-analyses have concluded that PPV23 confers protection against IPD but the duration of protection is unclear [11, 13-16]. We therefore investigated the PPV23 program in older adults in Taiwan over a study period of 8 years, from the pre- to the postPCV13 immunization era.

\section{Methods}

The PPV23 vaccination program and vaccine coverage A national PPV23 vaccination program aimed at the elderly ( $\geq 75$ years), with vaccines donated by the Formosa
Plastic Group, was implemented in 2008 [9]. In addition, during the study period seven counties/cities (Tainan, Yunlin, Taichung, and Lianjiang counties, and the cities of Taichung, Tainan, and Chiayi) introduced local, publicly funded PPV23 vaccination programs that provided free PPV23 immunization to residents 65-74 years of age.

\section{Data sources}

Data on IPD cases in patients of all ages in whom disease onset was between July 1, 2008, and June 30, 2016, were obtained from the national IPD surveillance system, a hospital laboratory- and case-based passive surveillance system for monitoring IPD established on October 15, 2007, by the Taiwan Centers for Disease Control [2, 17]. The IPD surveillance database contains demographic and clinical data, including IPD onset date and high-risk medical conditions (HRMCs, such as immunodeficiency/cancer, chronic obstructive pulmonary disease, congenital heart disease, splenectomy/asplenism, neurological disease, organ transplantation, congenital metabolic disorders, and other major illnesses) [2].

The PPV23 vaccination date was obtained from the National Immunization Information System (NIIS) database, described in previous studies $[2,18,19]$. The national vaccine registry is primarily designed to collate childhood vaccination data into a single web-based repository. However, it has been extended to include the registration of publicly funded PPV23 vaccination in the elderly and voluntary, self-paid childhood and adult vaccination. By linking the NIIS to the National Household Registration System, which includes all citizens with identifiers, we were able to calculate the coverage rate of PPV23 immunization in Taiwan for vaccine- targeted age groups.

\section{Statistical analysis}

Incidence rates of IPD were calculated per 100,000 inhabitants, and specific incidence rates by age groups $(\leq 5$, $6-64,65-74$, and $\geq 75$ years) and vaccine serotype. Incidence rate of serotype 19A-IPD was specifically characterized because it emerged after 7-valent PCV introduction and tends to result in more complicated pneumonia with empyema [6]. The rates were compared using relative risk (RR) and the 95\% confidence interval 
(95\% CI). Age-specific data on inhabitants in Taiwan were obtained from the Taiwan National Household Registration on a yearly basis. The Cochran-Armitage test was used to assess the trends in annual IPD incidence. Individuals were considered to be vaccinated if their PPV23 vaccination date was $\geq 14$ days before IPD onset. We excluded those received neither 2 doses of PPV23 nor PCV13 plus PPV23 from the study. Differences between vaccinated and unvaccinated patients were estimated using a chi-squared test or Fisher's exact test to compare proportions and by a linear regression model to compare continuous variables.

PPV23VE was calculated using two methods: the screening method and the indirect cohort (Broome) method. The screening method, described by Farrington $[10,20,21]$, is based on the comparison of the proportion of vaccinated cases with the proportion of the vaccinated population [21], and by its assumption in nature, instead of data on a control group, data on the whole population are used for contrast with vaccine coverage in the cases [22]. The VE was expressed as VE $=\frac{1-[P c(1-P p)]}{P p(1-P c)} * 100 \%$, where $P c=$ the proportion of cases who have been vaccinated and $P p=$ the proportion of the target population who have been vaccinated. Using logistic regression models, VE obtained by the screening method could control for confounding variables of age group (75-84 and all 85+) and sex when data on the vaccination coverage in each subgroup was available.

On the other hand, the indirect (Broome) cohort design used IPD cases caused by PPV23 vaccine types (VT) as the case group and IPD cases caused by non-PPV23 serotypes (ST) as the control group (non-cases, the comparison group) [10, 23]. The basic assumption in the indirect cohort design is that PPV23 vaccine provides no protection against and does not increase the risk of IPD caused by non-PPV23 ST. [10, 23-25]. VE was estimated by comparing the vaccination odds (compared to no PPV23 vaccination) of cases with controls and calculated as $(1-$ odds ratio $) \times 100 \%[10,23]$. Potential confounders, including sex, age, HRMC, and year of symptom onset, were adjusted by logistic regression. The statistical power of indirect cohort method would decrease as the vaccine coverage increases (>50\%) and fewer VT cases occur [23]. VE was also estimated for IPD caused by 1) 11 serotypes included in PPV23, but not found in PCV13 (PPV23-non PCV13 VT); 2) each serotype included in PPV23 that had been identified in at least 30 cases, in which other PPV23 vaccine serotypes were excluded for analysis. VE in preventing PPV23-serotype IPD, calculated according to the indirect cohort method, was also expressed as the number needed to vaccinate per case prevented [10, 24]. To estimate VE according to different intervals after PPV23 vaccination, the interval between IPD onset and the date of vaccination was categorized as $\leq 1$ year, $>1$ and $\leq 2$ years, $>2$ and $\leq 3$ years, $>3$ and $\leq 4$ years, $>4$ and $\leq 5$ years, and $\geq 5$ years. All analyses were conducted using SAS software (ver. 9.4; SAS Institute, Cary, NC, USA).

\section{Ethical statement}

The Taiwan CDC approved the protocol of this study and waived the requirement for written informed consent because of the study's retrospective design and the use of data from administrative databases, thus, involving minimal risk to study participants.

\section{Results}

\section{Incidence of IPD by age group and vaccine serotype}

Between July 2008 and June 2016, 5324 cases of IPD were identified. A decreasing trend of annual IPD incidence was significant across all age groups ( $p$ values for trend in each age group $<0.05$ ). Before the implementation of national PCV13 vaccination programs in children in 2013, the highest incidence of IPD in children $\leq 5$ years occurred in the year of July 2010-June 2011, and a decrease from a peak of 21.23 cases per 100, 000 inhabitants during July 2010-June 2011 to 6.14 per 100,000 inhabitants during July 2015-June 2016 was determined (Table 1). A comparison of the IPD incidence of July 2013-June 2016 and July 2008-June 2013 showed reductions of $73 \%$ (95\% CI: $68-77 \%)$ and $60 \%$ (95\% CI: 50-68\%) in PCV13 VT and serotype 19A, respectively. Non-PCV13 serotype (non-PCV13 ST) IPD increased from 1.88 per 100,000 inhabitants during July 2008-June 2013 to 3.38 per 100,000 inhabitants during July 2013June 2016 ( $R R=1.80$, 95\% CI: 1.39-2.32). The IPD incidence among individuals 6-64 years who were not covered by national pneumococcal vaccination programs was lower than in other age groups. Reductions in PCV13 VT and PPV23 VT of 29\% (95\% CI: 20-36\%) and 25\% (95\% CI: 17-33\%), respectively, were determined when the IPD incidence of July 2013-June 2016 was compared with that of July 2008-June 2013. The incidence of non-PCV13 ST IPD increased from 0.41 per 100,000 inhabitants during July 2008-June 2013 to 0.52 during July 2013-June 2016 ( RR = 1.27, 95\% CI: 1.091.47). For the age group 65-74 years, a comparison of the IPD incidence during the periods July 2013-June 2016 and July 2008-June 2013 showed reductions in PCV13 VT and PPV23 VT of 43\% (95\% CI: 31-53\%) and $40 \%$ (95\% CI: 28-50\%), respectively, together with a 38\% (95\% CI: 6-79\%) increase in non-PCV13 ST IPD. Among adults $\geq 75$ years, a $44 \%$ (95\% CI: 9-65\%) reduction of PPV23-non PCV13 VT IPD, a 36\% (95\% CI: 25$45 \%$ ) reduction of PCV13 VT, and a 36\% (95\% CI: $26-$ $45 \%$ ) reduction of PPV23 VT, occurred based on a comparison of July 2013-June 2016 with July 2008-June 


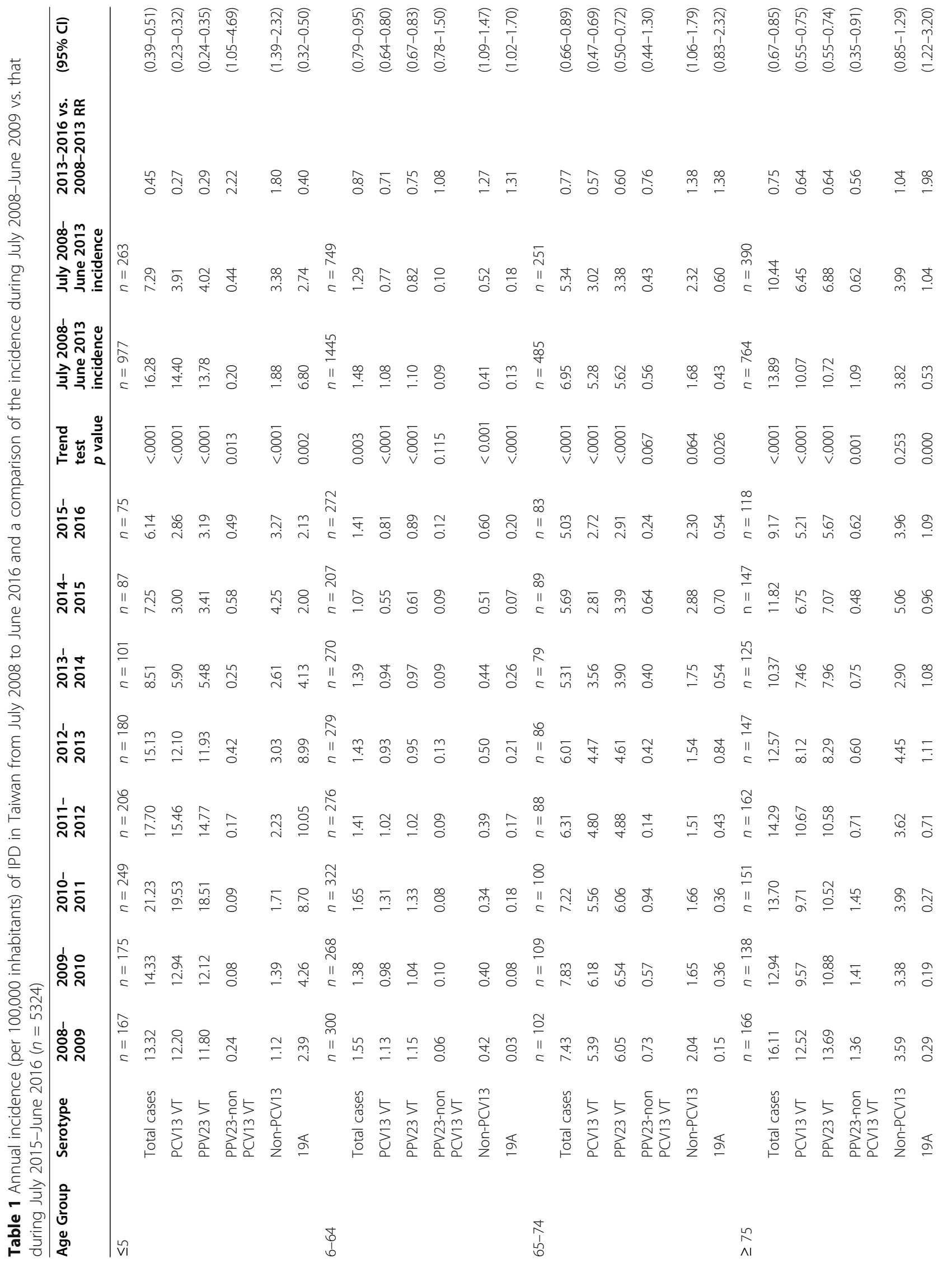




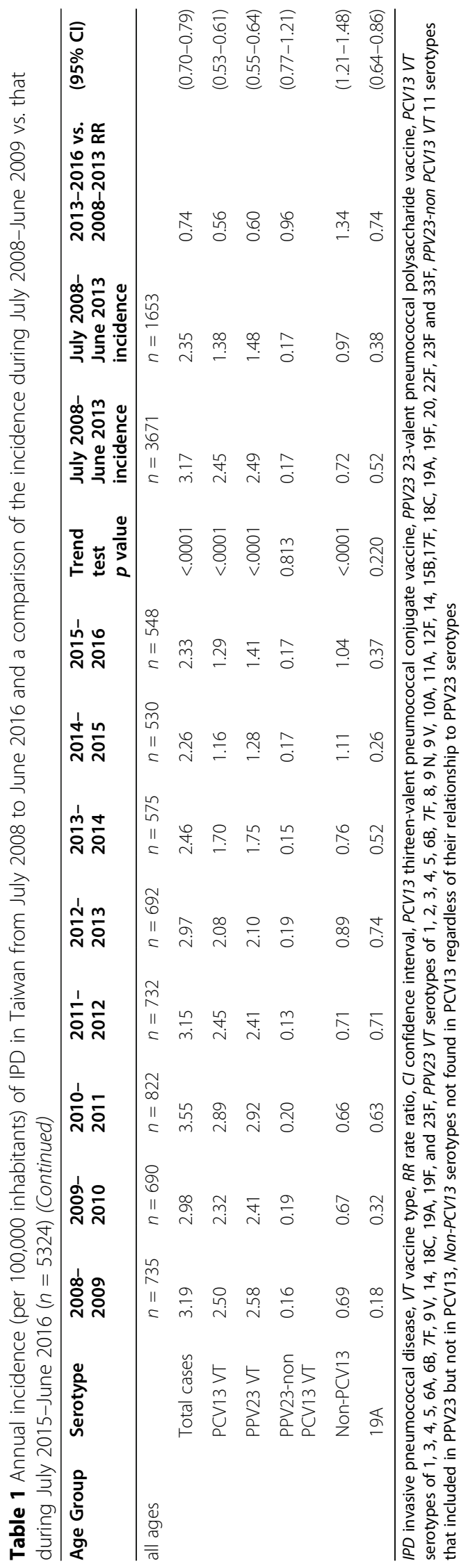


Table 2 Characteristics of IPD in patients $\geq 75$ years according to PPV23 status, July 2008 to June $2016(n=1154)$

\begin{tabular}{|c|c|c|c|c|c|c|c|}
\hline & Total & $\%$ & Unvaccinated & $\%$ & Vaccinated & $\%$ & $p$ value \\
\hline Total IPD cases & 1154 & 100 & 776 & 100 & 378 & 100 & \\
\hline Total IPD case serotyped & 1051 & 91.1 & 716 & 92.3 & 335 & 88.6 & 0.042 \\
\hline \multicolumn{8}{|l|}{ Gender } \\
\hline Female & 372 & 32.2 & 258 & 33.2 & 114 & 30.2 & 0.292 \\
\hline Male & 782 & 67.8 & 518 & 66.8 & 264 & 69.8 & 0.292 \\
\hline \multicolumn{8}{|l|}{ Onset age } \\
\hline Median (min max) & 83.1 & (75.0 106.6) & 82.8 & $(75.0 \sim 106.6)$ & 83.5 & (75.1 99.8) & 0.002 \\
\hline $75-84$ & 728 & 63.1 & 499 & 64.3 & 229 & 60.6 & 0.219 \\
\hline $85+$ & 426 & 36.9 & 277 & 35.7 & 149 & 39.4 & 0.219 \\
\hline \multicolumn{8}{|l|}{ Clinical manifestation } \\
\hline Sepsis & 621 & 53.8 & 417 & 53.7 & 204 & 54.0 & 0.941 \\
\hline Pneumonia & 776 & 67.2 & 513 & 66.1 & 263 & 69.6 & 0.239 \\
\hline Meningitis & 10 & 0.9 & 5 & 0.6 & 5 & 1.3 & 0.311 \\
\hline Others & 1081 & 93.7 & 720 & 92.8 & 361 & 95.5 & 0.108 \\
\hline \multicolumn{8}{|c|}{ Death within 30 days of IPD onset } \\
\hline $\mathrm{N}$ & 432 & 37.4 & 287 & 37.0 & 145 & 38.4 & 0.651 \\
\hline Age, Medium (min max) & 83.6 & (75.0 100.3) & 83.6 & $(75.0 \sim 100.3)$ & 84.1 & $(75.1 \sim 98.2)$ & 0.330 \\
\hline \multicolumn{8}{|c|}{ Days between onset to notification } \\
\hline Median (min max) & 5 & $(0.0 \sim 64.0)$ & 5 & $(0.0 \sim 64.0)$ & 5 & $(0 \sim 40.0)$ & 0.710 \\
\hline \multicolumn{8}{|l|}{ Onset season } \\
\hline 2008-2009 & 166 & 14.4 & 130 & 16.8 & 36 & 9.5 & 0.001 \\
\hline 2009-2010 & 138 & 12.0 & 100 & 12.9 & 38 & 10.1 & 0.164 \\
\hline 2010-2011 & 151 & 13.1 & 107 & 13.8 & 44 & 11.6 & 0.310 \\
\hline 2011-2012 & 162 & 14.0 & 107 & 13.8 & 55 & 14.6 & 0.727 \\
\hline 2012-2013 & 147 & 12.7 & 101 & 13.0 & 46 & 12.2 & 0.686 \\
\hline 2013-2014 & 125 & 10.8 & 81 & 10.4 & 44 & 11.6 & 0.538 \\
\hline $2014-2015$ & 147 & 12.7 & 83 & 10.7 & 64 & 16.9 & 0.003 \\
\hline $2015-2016$ & 118 & 10.2 & 67 & 8.6 & 51 & 13.5 & 0.011 \\
\hline Total IPD cases & 1154 & 100 & 776 & 100 & 378 & 100 & \\
\hline \multicolumn{8}{|c|}{ High risk medical conditions (HRMC) } \\
\hline With HRMC & 591 & 51.2 & 378 & 48.7 & 213 & 56.3 & 0.019 \\
\hline Immunodeficiency/cancer & 240 & 20.8 & 152 & 19.6 & 88 & 23.3 & 0.147 \\
\hline COPD & 214 & 18.5 & 133 & 17.1 & 81 & 21.4 & 0.079 \\
\hline Congenital heart disease & 6 & 0.5 & 3 & 0.4 & 3 & 0.8 & 0.400 \\
\hline Splectomy/Asplenism & 2 & 0.2 & 1 & 0.1 & 1 & 0.3 & 0.548 \\
\hline Others & 189 & 16.4 & 125 & 16.1 & 64 & 16.9 & 0.723 \\
\hline \multicolumn{8}{|l|}{ Serotypes } \\
\hline PCV13 VT & 795 & 68.9 & 554 & 71.4 & 241 & 63.8 & 0.009 \\
\hline PPV23 VT & 847 & 73.4 & 598 & 77.1 & 249 & 65.9 & $<.0001$ \\
\hline PPV23-non PCV13 VT & 83 & 7.2 & 69 & 8.9 & 14 & 3.7 & 0.001 \\
\hline
\end{tabular}

IPD invasive pneumococcal disease, COPD chronic obstructive pulmonary diseases, $V T$ vaccine type, $P C V 13$ thirteen-valent pneumococcal conjugate vaccine, $P P V 23$ 23-valent pneumococcal polysaccharide vaccine, PPV23-non PCV13 VT 11 serotypes that included in PPV23 but not in PCV13, that is serotypes of 2, 8, 9 N, 10A, $11 \mathrm{~A}, 12 \mathrm{~F}, 15 \mathrm{~B}, 17 \mathrm{~F}, 20,22 \mathrm{~F}$, and $33 \mathrm{~F}$ 


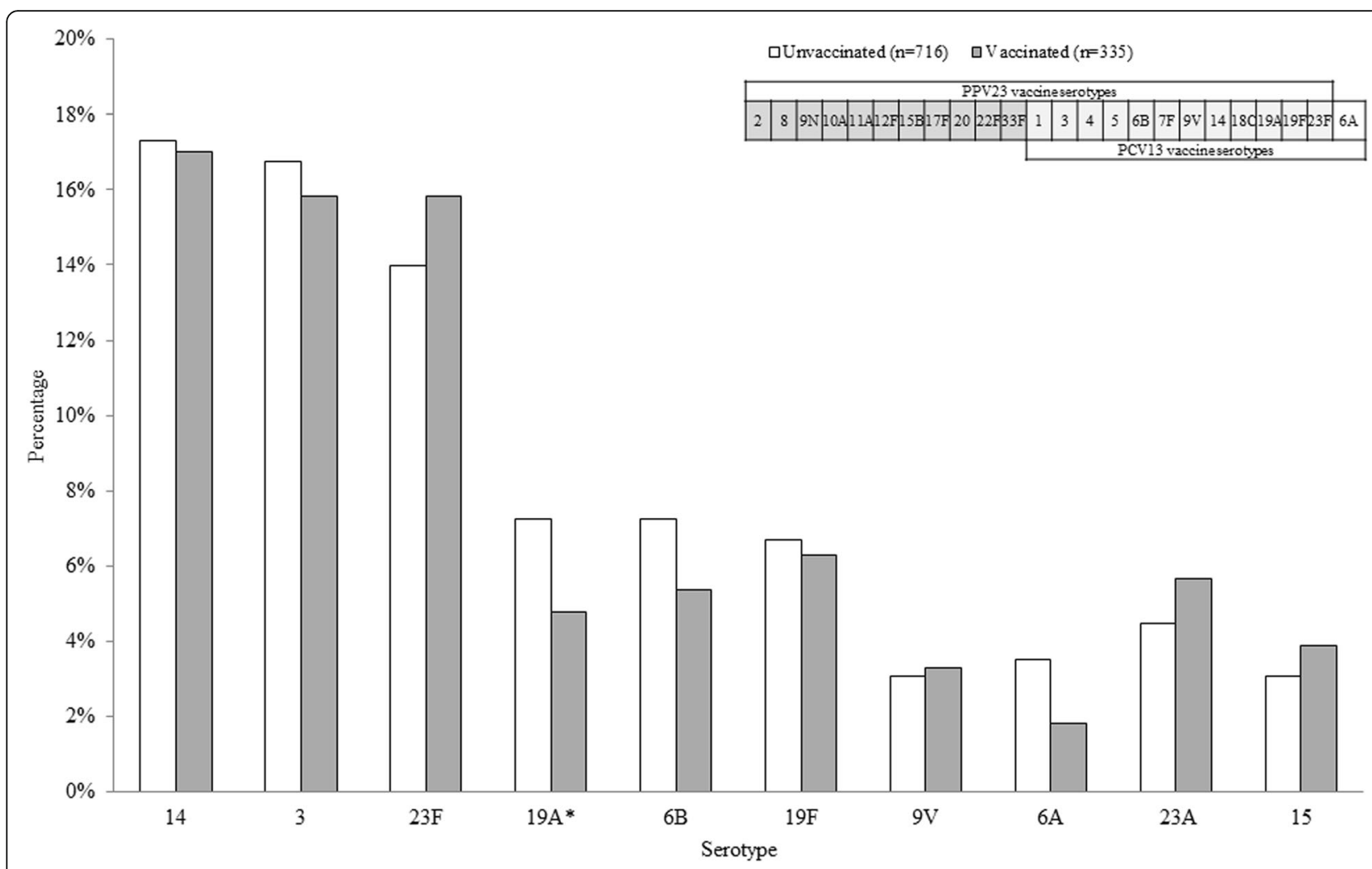

Fig. 1 Distribution of the serotypes identified in at least 30 cases of invasive pneumococcal disease in patients aged 75 years and over, stratified by PPV23 vaccination status, Taiwan, July 2008-June 2016, $n=1051$. The $Y$ axis indicates the percentage of serotype-specific IPD cases among PPV23 vaccinated and unvaccinated IPD patients, respectively. Among the IPD patients, serotypes 14, 3, 23F, 19A, 6B, 19F, and 9V were included in both PPV23 and PCV13. Serotype 6A is included in PCV13 but not in PPV23. Serotypes 23A and 15 (excluded 15B) are included in neither PPV23 nor PCV13. *: Serotype 19A differed significantly between vaccinated and unvaccinated individuals $(p<0.05)$

2013. Such decline of IPD in adults $\geq 75$ years after the introduction of PCV13 is likely due to a combination of direct effects from PPV23 and indirect effects from PCV13. The serotype percentage of PPV23-non PCV13 VT among the non-PCV13 ST was 37.8, 41.7, 36.4, 19.5, $13.5,25.7,9.5$, and $15.7 \%$, respectively, in adults $\geq 75$ years between July 2008-June 2009 and July 2015-June 2016 (Supplementary Table 1).

\section{Characteristics of IPD in adults $\geq \mathbf{7 5}$ years}

The incidence of IPD in adults $\geq 75$ years of age varied from 16.11 per 100,000 inhabitants during July 2008June 2009 to 9.17 per 100,000 inhabitants during July 2015-June 2016 ( $p$ for trend <.0001). Table 2 shows the characteristics of the 1154 IPD patients who were $\geq 75$ years old, the target population of the national PPV23 program. Among them, IPD affected more men (782 cases, $67.8 \%$ ) than women, with pneumonia as the main clinical manifestation (776 cases, 67.2\%). PPV23 had been administered to 378 individuals (32.8\%) and 591 (51.2\%) had HRMC. Serotyping was available for 1051 patients (91.1\%). The proportion of PPV23 serotypes was $65.9 \%$ in the vaccinated and $77.1 \%$ in the unvaccinated. Vaccinated patients were older at the age of IPD onset $(p<0.05)$, presented with more HRMCs ( $p$ $<0.05$ ), and had a lower proportion of PPV23-serotype IPD $(p<0.001)$ than unvaccinated patients. As shown in Fig. 1, the most common pneumococcal serotypes identified in adults $\geq 75$ years were: 14 (181 patients, $17.2 \%$ ), 3 (173 patients, 16.5\%), 23F (153 patients, 14.6\%), 6B (70 patients, 6.7\%), 19F (69 patients, 6.6\%), and 19A (68 patients, $6.5 \%)$, respectively. Of these, the only serotype that significantly differed between vaccinated and unvaccinated IPD patients was 19A $(p<0.05)$.

\section{Vaccine uptake}

Supplementary Table 2 shows the accumulated vaccine coverage of the population vaccinated with the PPV23 vaccine by June 30,2016 , according to the age groups $65-74,75-84$ and $\geq 85$ years. The data are nationally and regionally based and were calculated from NIIS data. National PPV23 coverage for people $\geq 75$ years of age was $41.9 \%$. Vaccine coverage was higher in the seven counties/cities than in other counties/cities for the age groups $65-74$ and $\geq 75$ years. In order to validate the PPV23 vaccination records for elderly adults in NIIS, an 
Table 3 Vaccine effectiveness of PPV23 in adults $\geq 75$ years, estimated by the screening method, July 2008 to June 2016

\begin{tabular}{|c|c|c|}
\hline IPD & VE & $(95 \% \mathrm{Cl})$ \\
\hline \multicolumn{3}{|l|}{ All serotypes } \\
\hline Crude VE & $32.4 \%$ & (23.6 to 40.2$)$ \\
\hline Adjusted by gender & $33.2 \%$ & (24.4 to 40.9 ) \\
\hline Adjusted by age and gender & $33.9 \%$ & (25.2 to 41.5$)$ \\
\hline \multicolumn{3}{|l|}{ Death within 30 days of IPD onset } \\
\hline Crude VE & $29.9 \%$ & (14.4 to 42.6$)$ \\
\hline Adjusted by gender & $30.8 \%$ & (15.5 to 43.4$)$ \\
\hline Adjusted by age and gender & $32.5 \%$ & (17.5 to 44.7$)$ \\
\hline \multicolumn{3}{|l|}{ PPV23 VT } \\
\hline Crude VE & $42.2 \%$ & (33.0 to 50.2 ) \\
\hline Adjusted by gender & $42.9 \%$ & (33.8 to 50.7 ) \\
\hline Adjusted by age and gender & $43.4 \%$ & (34.4 to 51.2 ) \\
\hline \multicolumn{3}{|l|}{ PPV23-non PCV13 VT } \\
\hline Crude VE & $71.8 \%$ & (50.0 to 84.2$)$ \\
\hline Adjusted by gender & $72.1 \%$ & (50.5 to 84.3 ) \\
\hline Adjusted by age and gender & $72.6 \%$ & (51.4 to 84.6$)$ \\
\hline \multicolumn{3}{|l|}{ PCV13 (without 6A) VT } \\
\hline Crude VE & $38.4 \%$ & (28.1 to 47.1 ) \\
\hline Adjusted by gender & $39.1 \%$ & (29.0 to 47.8 ) \\
\hline Adjusted by age and gender & $39.6 \%$ & (29.5 to 48.2 ) \\
\hline
\end{tabular}

IPD invasive pneumococcal disease, $V E$ vaccine effectiveness, $C I$ confidence interval, $V T$ vaccine type, PPV23 23-valent pneumococcal polysaccharide vaccine, $P C V 13$ thirteen-valent pneumococcal conjugate vaccine, $P P V 23$-non $P C V 13$ VT 11 serotypes that included in PPV23 but not in PCV13, that is serotypes of $2,8,9 \mathrm{~N}, 10 \mathrm{~A}, 11 \mathrm{~A}, 12 \mathrm{~F}, 15 \mathrm{~B}, 17 \mathrm{~F}, 20,22 \mathrm{~F}$, and $33 \mathrm{~F}$

estimation of $80.6 \%$ of 853,460 doses of PPV23 procurement by the government between July 2008 to June 2016 was registered with vaccinee information identified in the NIIS database, including 570,665 and 117,295 individuals immunized with PPV23 vaccination at age of $\geqq 75$ years and 65-74 years, respectively. The missing PPV23 records could be possibly explained by the doses that have not been used, doses that have been wasted, or a truly unregistered PPV23 vaccination records.

\section{Vaccine effectiveness}

PPV23VE in adults $\geq 75$ years as estimated by the screening method is shown in Table 3. The sex- and ageadjusted VE was 33.9\% (95\% CI: 25.2-41.5\%) in preventing all-serotype IPD, 32.5\% (95\% CI: $17.5-44.7 \%)$ in preventing death within 30 days of IPD onset, $43.4 \%$ (95\% CI: 34.4-51.2\%) in preventing IPD caused by PPV23 VT, 72.6\% (95\% CI: 51.4-84.6\%) in preventing IPD caused by PPV23-non PCV13 VT, and 39.6\% (95\% CI: 29.5-48.2\%) in preventing IPD caused by PCV13 (excluding 6A) VT.

The effectiveness of PPV23 as estimated by the indirect cohort method was 39.0\% (95\% CI: 15.5-55.9\%; Table 4), which corresponded to a number needed to vaccinate of 13,173 (95\% CI: 10,093-33,146) per PPV23serotype IPD case prevented. VE was not significant in subgroups of patients $\geq 85$ years $(33.9 \%$; 95\% CI: -15.2 to 62.1$)$ and in patients with HRMCs $(20.4 \%$; $95 \%$ CI: 24.5 to 49.1$)$. VE increased when only 11 serotypes included in PPV23 but not in PCV13 were considered. According to the indirect cohort method (Table 4), VE in preventing PPV23-non PCV13 VT was 71.5\% (95\% CI: 44.2-85.4\%), which was similar to the VE estimated by the screening method (Table 3). For the PPV23 serotypes identified in at least 30 cases, a significant VE was determined for serotypes 15B, 19A, 6B, 3 and 14 but not for serotypes $23 \mathrm{~F}$ and $19 \mathrm{~F}$ (Table 4). Supplementary Table 3 shows VE against IPD from July 2008 to June 2012, by excluding the PCV13 program period, and a higher point estimate of VE was observed for PPV23 VT (VE=55.1, 95\% CI: 27.2-72.3\%) and PPV23-non PCV13 VT (VE=79.8, 95\% CI: 47.2-92.3\%), respectively, while comparing with VE estimated against the same serotypes during the period July 2018-June 2016.

\section{Differential vaccine effectiveness of PPV23 over time}

We also assessed VE according to different intervals after vaccination. The adjusted PPV23VE in adults $\geq 75$ years according to the indirect cohort method was $44.9 \%$ (95\% CI: 20.8-61.7) within 5 years of vaccination and 15.5\% (95\% CI: -47.1 to 51.4 ) thereafter (Table 4). The adjusted PPV23VE in adults $\geq 75$ years of age was $73.7 \%$ (39.0-88.7) when $\leq 1$ year had elapsed, $64.4 \%$ (29.2-82.0) when $>1$ year but $\leq 2$ years had elapsed, $48.9 \%$ (95\% CI: -1.6 to 74.3$)$ when $>2$ years but $\leq 3$ years had elapsed, 19.2\% (95\% CI: -61.3 to 59.5 ) when $>3$ years but $\leq 4$ years had elapsed, and $-3.4 \%$ ((95\% CI: -144.9 to 56.3$)$ when $>4$ years but $\leq 5$ years had elapsed (Table 5 ). The wide interval for the $>4$ and $\leq 5$ years might be explained by the smaller sample size, compared to other categories of time since vaccination.

\section{Discussion}

For countries with long-term laboratory-based systems for monitoring IPD, linking IPD surveillance with valid pneumococcal immunization records facilitates the evaluation of vaccination programs pre- and postimplantation from a public health perspective [1]. In determinations of $\mathrm{VE}$, the results of effectiveness studies might be at risk of bias due to the impact of patient health and the short study period, as was the case in a previously published nationwide study of PPV23 effectiveness in Taiwan. In contrast, our study used data from subsequent years in similar adult populations and drew on different observational methodologies $[9,16]$. The results showed that with respect to IPD and 30-day fatality, PPV23VE was lower than expected when the study period was extended to 8 years. Based on observational 
Table 4 Effectiveness of PPV23 in patients $\geq 75$ years of age, estimated by the indirect cohort (Broome) method, July 2008 to June $2016(n=1051)$

\begin{tabular}{|c|c|c|c|c|c|c|}
\hline IPD & Cases & Controls & Crude VE (\%) & $95 \% \mathrm{Cl}$ & Adjusted VE (\%) & $95 \% \mathrm{Cl}$ \\
\hline Serotypes PPV23 & 847 & 204 & 42.9 & (21.7 to 58.3 ) & $39.0^{\mathrm{a}}$ & (15.5 to 55.9$)$ \\
\hline $75-84$ & 540 & 133 & 44.9 & (18.5 to 62.8$)$ & $42.4^{\mathrm{b}}$ & (13.7 to 61.6$)$ \\
\hline $85+$ & 307 & 71 & 39.5 & $(-2.5$ to 64.3$)$ & $33.9^{b}$ & $(-15.2$ to 62.1$)$ \\
\hline Female & 263 & 82 & 46.1 & (9.5 to 68.0$)$ & $38.7^{c}$ & $(-4.9$ to 64.2$)$ \\
\hline Male & 584 & 122 & 42.5 & (14.3 to 61.4$)$ & $38.0^{c}$ & (6.4 to 59.0$)$ \\
\hline Without HRMC & 390 & 95 & 57.6 & (32.3 to 73.4 ) & $56.5^{d}$ & (29.6 to 73.1$)$ \\
\hline With HRMC & 425 & 108 & 27.5 & $(-11.7$ to 52.9$)$ & $20.4^{d}$ & $(-24.5$ to 49.1$)$ \\
\hline$>5$ years after vaccination & 668 & 143 & 44.7 & (9.1 to 66.4 ) & $15.5^{\mathrm{a}}$ & $(-47.1$ to 51.4$)$ \\
\hline$\leq 5$ years after vaccination & 777 & 179 & 42.1 & (17.7 to 59.2$)$ & $44.9^{\mathrm{a}}$ & (20.8 to 61.7 ) \\
\hline Serotypes PPV23-non PCV13 & 83 & 204 & 72.2 & (47.3 to 85.3 ) & $71.5^{\mathrm{a}}$ & (44.2 to 85.4 ) \\
\hline Serotype PCV13 (without 6A) & 764 & 204 & 39.0 & $(16.3 \circ 55.6)$ & $35.3^{\mathrm{a}}$ & (10.1 to 53.4 ) \\
\hline serotype 3 & 173 & 204 & 39.4 & (7.2 to 60.4 ) & $36.8^{\mathrm{a}}$ & (0.4 to 59.9 ) \\
\hline Serotype14 & 181 & 204 & 36.9 & (4.1 to 58.5 ) & $38.8^{\mathrm{a}}$ & (4.1 to 61.0 ) \\
\hline serotype $23 F$ & 153 & 204 & 27.3 & $(-12.2$ to 52.9$)$ & $11.3^{\mathrm{a}}$ & $(-42.1$ to 44.7$)$ \\
\hline serotype 19F & 69 & 204 & 40.0 & $(-7.6$ to 66.5$)$ & $34.9^{\mathrm{a}}$ & ( -21.3 to 65.1$)$ \\
\hline serotype 19A & 68 & 204 & 57.8 & (21.1 to 77.4 ) & $56.3^{\mathrm{a}}$ & (16.1 to 77.2 ) \\
\hline serotype 6B & 70 & 204 & 52.5 & (13.1 to 74.0 ) & $51.9^{\mathrm{a}}$ & (8.1 to 74.8 ) \\
\hline serotype 15B & 23 & 204 & 79.4 & (28.5 to 94.1 ) & $80.6^{\mathrm{a}}$ & (28.6 to 94.7 ) \\
\hline serotype $9 \mathrm{~V}$ & 33 & 204 & 31.4 & $(-49.0$ to 68.4$)$ & $31.3^{\mathrm{a}}$ & (-60.4 to 70.6$)$ \\
\hline
\end{tabular}

VE vaccine effectiveness, $C l$ confidence interval, $H R M C$ high-risk medical conditions, $P P V 23$ 23-valent pneumococcal polysaccharide vaccine, $P C V 13$ thirteen-valent pneumococcal conjugate vaccine. Serotypes PPV23-non PCV13 VT: 11 serotypes that included in PPV23 but not in PCV13, that is serotypes of 2, 8, 9 N, 10A, 11A, $12 \mathrm{~F}, 15 \mathrm{~B}, 17 \mathrm{~F}, 20,22 \mathrm{~F}$, and $33 \mathrm{~F}$

${ }^{\mathrm{a}}$ Adjusted for age group, sex, presence of HRMC, and onset year; ${ }^{\mathrm{b}}$ Adjusted for sex, presence of HRMC, and onset year; ${ }^{\mathrm{C} A d j u s t e d}$ for age group, presence of HRMC, and onset year; ${ }^{d}$ Adjusted for age group, sex, and onset year

studies, the estimated PPV23VE against IPD in older adults or adults with conditions associated with an increased risk of IPD was $27-76 \%[9,11,13,16,24,26$, 27]. Previously reported estimates of VE have differed, most likely because of the different methods used and the different study periods in the estimations [14, 27]. The point estimate of PPV23VE in this study was within the $95 \% \mathrm{CI}$ of the estimated VE reported in a Cochrane review of non-random controlled trials in adults $(\mathrm{VE}=$ 52, 95\% CI: 39-63\%) [13].
The decline in IPD among adults $\geq 75$ years from preto post-PCV13 immunization era is likely due to a combination of direct effects from PPV23 and indirect effects from PCV13, which are epidemiologically challenging to tease apart. Therefore, we applied the indirect cohort method to evaluate PPV23VE during the period July 2008-June 2012 when national PCV13 program was not introduced in children. PPV23VE for elderly seemed higher in the period of July 2008-June 2012 (VE=55.1, 95\% CI: $27.2-72.3 \%$ ) than in the period of July 2008-

Table 5 Effectiveness of PPV23 in patients $\geq 75$ years of age at different intervals after vaccination, estimated by the indirect cohort (Broome) method, July 2008 to June 2016 ( $n=1051)$

\begin{tabular}{|c|c|c|c|c|c|c|}
\hline Time since PPV23 vaccination & Cases & Controls & Crude VE (\%) & $95 \% \mathrm{Cl}$ & Adjusted VE (\%) & $95 \% \mathrm{Cl}$ \\
\hline No vaccination & 598 & 118 & Ref. & & Ref. & \\
\hline$\leq 1$ years & 29 & 11 & 48.0 & $(-7.0$ to 74.7$)$ & 73.7 & (39.0 to 88.7 ) \\
\hline$>1$ and $\leq 2$ years & 41 & 15 & 46.1 & $(-0.6$ to 71.1$)$ & 64.4 & (29.2 to 82.0$)$ \\
\hline$>2$ and $\leq 3$ years & 37 & 14 & 47.9 & (0.5 to 72.7$)$ & 48.9 & $(-1.6$ to 74.3$)$ \\
\hline$>3$ and $\leq 4$ years & 46 & 13 & 30.2 & $(-33.3$ to 63.4$)$ & 19.2 & $(-61.3$ to 59.5$)$ \\
\hline$>4$ and $\leq 5$ years & 26 & 8 & 35.9 & $(-45.1$ to 71.7$)$ & -3.4 & $(-144.9$ to 56.3$)$ \\
\hline$>5$ years & 70 & 25 & 44.7 & (9.1 to 66.4$)$ & 15.5 & $(-47.1$ to 51.4$)$ \\
\hline
\end{tabular}

PPV23 23-valent pneumococcal polysaccharide vaccine, Ref reference, $V E$ vaccine effectiveness, $C l$ confidence interval 
June 2016 (VE=39.0, 95\% CI: 15.5-50.9\%). We speculated that as the indirect protection emerged following PCV13 introduction, PPV23 unvaccinated elderly could benefit from lowering their risk of acquiring IPD caused by 12 serotypes common to PCV13 and PPV23, which might bias the PPV23VE estimation to be lower. On the other hand, VE waning since time of vaccination should possibly be considered.

In addition, in our study, PPV23VE was higher against IPD caused by 11 serotypes that included in PPV23 but not in PCV13 (PPV23-non PCV13 VT) than against PPV23 serotype-IPD, as also found in a Spanish study [10]. Such findings were observed using either the screening method or the indirect cohort method. This difference may be due to a differential effectiveness against different serotypes $[10,28]$. However, other possible causes remain to be explored in future studies [10,23, 28, 29].

The validity of VE estimation using the screening method could be influenced by the completeness of PPV23 immunization recorded in the NIIS. Although the accurate PPV23 vaccination coverage for IPD cases and elderly population might be greater than 32.8 and $41.9 \%$, respectively, it may not result in very low or very high $P p$ and $P c$ to bias VE estimation [30, 31]. PPV23VE estimates by the indirect cohort method would possibly bias if the PPV23 vaccinated and unvaccinated individuals are not at the same risk of non-PPV23 ST infections $[10,23]$. Unlike PCV-induced serotype replacement, PPV23 would not drive the increase of non-PPV23 ST among vaccinated and unvaccinated elderly.

The adjusted VE determined by the screening method was slightly higher than the estimated VE obtained using the Broome method, perhaps because the screening method does not allow for the control of confounding factors, which can result in an overestimation of VE [21]. Moreover, the study population applied in the Broome method consisted of all patients who had developed IPD (case-case comparison approach) [32], in whom the proportion of underlying disease may have been higher than in community-dwelling elderly. Differences in the immune responses to vaccination of the case-case population compared to the communitydwelling elderly population may lead to a lower VE estimate $[16,33]$.

Following the 2006 recommendation by the $\mathrm{WHO}$ of a routine PCV-based immunization program in children, the IPD incidence caused by PCV VT gradually declined not only in vaccinated (direct protection) but also in unvaccinated (indirect protection) age groups [3, 34, 35]. From before (July 2008-June 2013) to after (July 2013June 2016) the implementation of a children's PCV13 program in Taiwan, the highest age-specific annual IPD incidence shifted from age $2-4$ years to $\geq 75$ years, with a decreasing trend in the incidence of IPD related to
PCV13 VT across all age groups. Previous studies reported an increase in IPD, due to non-PCV serotypes or serotype replacement, roughly 3-4 years after the introduction of a PCV program [36-38]. The increasing trend in the incidence of non-PCV13 ST IPD determined in this study involved all age groups except adults $\geq 75$ years, i.e., the target population of the national PPV 23 program. Whether serotype replacement occurs over different time intervals in different age groups or is influenced by the different serotype distributions among age groups, especially for those covered by the PPV23 vaccination, remains to be investigated. Long-term surveillance of IPD, nasal carriage, and non-bacteremic pneumococcal pneumonia will be crucial in the monitoring of serotype replacement and in ascertaining whether pneumococcal vaccines offer direct or indirect protection against the incidence of disease over the long term [36].

Vaccine protection at the population level can be rapidly estimated using the screening method whereas the duration of PPV23 protection at an individual level could be estimated by a stratified analysis of time since vaccination using the indirect cohort method $[10,11$, 21]. In this study, the change in PPV23VE since the time of vaccination, measured at an interval of every 1-year between IPD onset and the date of vaccination, could be considered a diagnostic indication of waning VE $[10,15$, 39]. The wide confidence interval and biased point estimate of VE in the subgroup of the interval of $>4$ and $\leq$ 5 years could be possibly explained by the small sample size [40]. A study from England and Wales reported a PPV23VE of $48 \%$ against IPD within 2 years of vaccination for adults $\geq 65$ years, as determined by the indirect cohort method, but VE waned and became insignificant beyond 5 years [28]. In the effectiveness of pneumococcal vaccination against community-acquired pneumonia, acute myocardial infarction and stroke (CAPAMIS) study, effectiveness estimates became higher and significant after patients vaccinated $>60$ months previously were excluded [41]. The differential VE against PPV23serotype IPD observed over time in our study was in agreement with a previous report but the lack of significance of the 2- to 5-year interval may have been due to the lower statistical power resulting from stratification of the variable "time since vaccination" [10].

PPV23 is a T-cell-independent vaccine that lacks a mechanism for long-term boosting of the immune response [42]. While PPV23 revaccination may result in a significant and sustained antibody responses in adults, including the elderly [43,44], it is recommended only for those with an increased risk of IPD and no sooner than 5 years after the first dose [45]. However, insufficient data regarding clinical benefit, the degree and duration of protection, and safety have hindered a 
routine recommendation of revaccination [46]. In the USA, as in Taiwan, a single dose of PPV23 is recommended for all adults $\geq 65$ years of age regardless of the previous history of PPV23 [3, 46]. In the elderly and in risk groups in middle-high income countries, PCV13 and PPV23 immunization is recommended to confer better and longer protection [42, 47]. In fact, in many countries, PPV23 is recommended as an effective and/or cost-effective vaccine covering a broad array of the serotypes implicated in IPD in older adults $[10,45,48]$.

Our study had several limitations beyond its observational design and the residual confounders. First, although the coverage of hospitals enrolled in IPD surveillance in Taiwan should have been $100 \%$, detection bias may have occurred for cases identified using this passive surveillance system, thereby affecting the representativeness of the data $[2,17]$. Second, PPV23 vaccine records in the NIIS database were prospectively collected after the endorsement of a publicly funded PPV vaccination program for elderly individuals. Information on missing vaccination status was not determined by the occurrence of IPD outcome. Therefore, the probability of misclassification of vaccinated to be unvaccinated might be non-differential between cases and controls in the indirect cohort method. Third, IPD surveillance has been started with the implementation of the national PPV23 vaccination program in the elderly. We could not evaluate the impact of PPV23 program from pre- to post-PPV23 vaccination program by changes of IPD incidence. Fourth, influenza vaccination status is an important covariate that should be considered in evaluations of PPV23VE in the elderly [49]. However, the NIIS database contains limited annual individual influenza vaccine records and it was not possible to determine seasonal influenza status as covariate information for the elderly population in our study.

\section{Conclusions}

In conclusion, our results demonstrated a decrease in IPD incidence among all age groups across eight seasons covering the pre- to post-national PCV13 immunization era in Taiwan, accompanied by an increased trend of non-PCV13 ST IPD. The decline in IPD among adults $\geq$ 75 years is likely due to a combination of direct effects from PPV23 and indirect effects from PCV13. Using the indirect cohort method, PPV23 did confer moderate protection against PPV23-serotype IPD in adults $\geq 75$ years before national PCV13 immunization program implemented in children.

\section{Supplementary Information}

The online version contains supplementary material available at https://doi. org/10.1186/s12879-020-05721-0.
Additional file 1: Supplementary Table 1. Serotype percentage of IPD in Taiwan from July 2008 to June 2016, stratified by age group.

Additional file 2: Supplementary Table 2. Accumulated coverage of PPV23 in older adults, stratified by counties and age groups in Taiwan.

Additional file 3: Supplementary Table 3. Effectiveness of PPV23 in patients $\geq 75$ years of age, estimated by the indirect cohort (Broome) method, July 2008 to June $2012(n=581)$.

\section{Abbreviations}

IPD: Invasive pneumococcal disease; PCV: Pneumococcal conjugate vaccine; VE: Vaccine effectiveness; PPV23: 23-valent pneumococcal polysaccharide vaccine; PCV13: 13-valent PCV; WHO: World Health Organization;

HRMC: High-risk medical condition; NIIS: National Immunization Information System; RR: Relative risk; PC: Proportion of cases who have been vaccinated; Pp: Proportion of the target population who have been vaccinated;

VT: Vaccine type; ST: Serotype; CAPAMIS: Community-acquired pneumonia, acute myocardial infarction and stroke

\section{Acknowledgements}

The authors would like to thank all of the public health workers and health care providers who promoted the pneumococcal vaccination program and participated in invasive pneumococcal disease control and prevention in Taiwan.

\section{Authors' contributions}

WJS and CHY were responsible for the study concept and design; WJS, HYL, CSC, ETW, and CHY were responsible for acquisition of data; WJS and PHC were responsible for analysis of the data; WJS, PHC, LYC, and CHY were responsible for interpretation of data; WJS were responsible for drafting the manuscript; WJS, PHC, LYC, and CHY were responsible for critical revision of the manuscript for important intellectual content; HYL and ETW were responsible for administrative, technical, or logistic support. CSC participated in the laboratory diagnosis. All authors read and approved the final manuscript.

\section{Funding}

The study is not supported by any funding resources.

\section{Availability of data and materials}

The datasets used and analyzed during the current study are not publicly available due to it contains individual data and maintained by the public health authorities but are available from the corresponding author on reasonable request.

\section{Ethics approval and consent to participate}

The Taiwan Centers for Disease Control approved the protocol of this study by its Institutional Review Board (IRB, no.104206) and waived the requirement for written informed consent because of the study's retrospective design and the use of data from administrative databases, thus, involving minimal risk to study participants.

Consent for publication

Not applicable.

\section{Competing interests}

The authors declare that we have no conflicts (financial, professional, or personal) relevant to the manuscript.

\section{Author details}

${ }^{1}$ Division of Acute Infectious Diseases, Centers for Disease Control, Ministry of Health and Welfare, 6, Linsen South Road, Taipei 100, Taiwan, Republic of China. ${ }^{2}$ Department of Pediatrics, National Taiwan University Hospital, Taipei, Taiwan, Republic of China. ${ }^{3}$ Center for Prevention and Treatment of Occupational Injury and Diseases, Taipei Veterans General Hospital, Taipei, Taiwan, Republic of China. ${ }^{4}$ Division of Clinical Toxicology and Occupational Medicine, Department of Medicine, Taipei Veterans General Hospital, Taipei, Taiwan, Republic of China. 
Received: 22 April 2020 Accepted: 18 December 2020 Published online: 10 January 2021

\section{References}

1. Publication WHO. Pneumococcal vaccines WHO position paper - 2012 recommendations. Vaccine. 2012;30(32):4717-8.

2. Su WJ, Lo HY, Chang CH, Chang LY, Chiu CH, Lee Pl, Lu CY, Hsieh YC, Lai MS, Lin TY. Effectiveness of pneumococcal conjugate vaccines of different valences against invasive pneumococcal disease among children in Taiwan: a Nationwide study. Pediatr Infect Dis J. 2016;35(4):e124-33.

3. Su WJ, Yang $\mathrm{CH}$. Control and prevention of invasive pneumococcal disease in Taiwan: current achievements and future challenges. J Formos Med Assoc. 2019;118:961-4.

4. ABCs Report: Streptococcus pneumoniae, 2000 [https://www.cdc.gov/abcs/ reports-findings/survreports/spneu00.html].

5. ABCs Report: Streptococcus pneumoniae, 2016 [https://www.cdc.gov/abcs/ reports-findings/survreports/spneu16.html].

6. Lu CY, Chiang CS, Chiu CH, Wang ET, Chen YY, Yao SM, Chang LY, Huang LM, Lin TY, Chou JH. Successful control of Streptococcus pneumoniae 19A replacement with a catch-up primary vaccination program in Taiwan. Clin Infect Dis. 2019;69(9):1581-7.

7. Pilishvili T, Whitney CG. Use of data to drive pneumococcal conjugate vaccine policy. Lancet Infect Dis. 2018;18(4):366-8.

8. Ingels $\mathrm{H}$, Rasmussen J, Andersen PH, Harboe ZB, Glismann S, Konradsen H, Hoffmann S, Valentiner-Branth P, Lambertsen L. Danish pneumococcal surveillance collaboration $\mathrm{G}$ : impact of pneumococcal vaccination in Denmark during the first 3 years after PCV introduction in the childhood immunization programme. Vaccine. 2012;30(26):3944-50.

9. Tsai YH, Hsieh MJ, Chang CJ, Wen YW, Hu HC, Chao YN, Huang YC, Yang $C T$, Huang CC. The 23-valent pneumococcal polysaccharide vaccine is effective in elderly adults over 75 years old-Taiwan's PPV vaccination program. Vaccine. 2015;33(25):2897-902.

10. Gutierrez Rodriguez M, Ordobas Gavin M, Garcia-Comas L, Sanz Moreno J, Cordoba Deorador E, Lasheras Carbajo M, Taveira Jimenez J, Martin Martinez F, Iniesta Fornies D, Arce Arnaez A. Effectiveness of 23-valent pneumococcal polysaccharide vaccine in adults aged 60 years and over in the Region of Madrid, Spain, 2008-2011. Euro Surveill. 2014;19(40):20922.

11. Kraicer-Melamed H, O'Donnell S, Quach C. The effectiveness of pneumococcal polysaccharide vaccine 23 (PPV23) in the general population of 50 years of age and older: a systematic review and meta-analysis. Vaccine. 2016;34(13):1540-50.

12. Duclos $P$, Durrheim DN, Reingold AL, Bhutta ZA, Vannice $K$, Rees $H$. Developing evidence-based immunization recommendations and GRADE. Vaccine. 2012;31(1):12-9.

13. Moberley S, Holden J, Tatham DP, Andrews RM. Vaccines for preventing pneumococcal infection in adults. Cochrane Database Syst Rev. 2013;1: CD000422.

14. Huss A, Scott P, Stuck AE, Trotter C, Egger M. Efficacy of pneumococcal vaccination in adults: a meta-analysis. CMAJ. 2009;180(1):48-58.

15. Tin Tin Htar M, Stuurman AL, Ferreira G, Alicino C, Bollaerts K, Paganino C, Reinert RR, Schmitt HJ, Trucchi C, Vestraeten T, et al. Effectiveness of pneumococcal vaccines in preventing pneumonia in adults, a systematic review and meta-analyses of observational studies. PLoS One. 2017;12(5): e0177985.

16. Falkenhorst $\mathrm{G}$, Remschmidt $\mathrm{C}$, Harder $\mathrm{T}$, Hummers-Pradier $\mathrm{E}$, Wichmann $\mathrm{O}$, Bogdan C. Effectiveness of the 23-Valent pneumococcal polysaccharide vaccine (PPV23) against pneumococcal disease in the elderly: systematic review and meta-analysis. PLoS One. 2017;12(1):e0169368.

17. Chiang CS, Chen YY, Jiang SF, Liu DP, Kao PH, Teng HJ, Kuo TL, Yao SM, Tseng LR, Wang $Y L$, et al. National surveillance of invasive pneumococcal diseases in Taiwan, 2008-2012: differential temporal emergence of serotype 19A. Vaccine. 2014;32(27):3345-9.

18. Liu DP, Wang ET, Pan YH, Cheng SH. Innovative applications of immunisation registration information systems: example of improved measles control in Taiwan. Euro Surveill. 2014;19(50):20994.

19. Su WJ, Chen SF, Yang CH, Chuang PH, Chang HF, Chang MH. The Impact of Universal Infant Hepatitis B Immunization on reducing the Hepatitis B Carrier Rate in Pregnant Women. J Infect Dis. 2019;220(7):1118-26. https:// doi.org/10.1093/infdis/jiy706.

20. Farrington CP. Estimation of vaccine effectiveness using the screening method. Int J Epidemiol. 1993;22(4):742-6.
21. Orenstein WA, Bernier RH, Dondero TJ, Hinman AR, Marks JS, Bart KJ, Sirotkin B. Field evaluation of vaccine efficacy. Bull World Health Organ. 1985;63(6):1055-68.

22. Rodrigues LC, Smith PG. Use of the case-control approach in vaccine evaluation: efficacy and adverse effects. Epidemiol Rev. 1999;21(1):56-72.

23. De Serres G, Pilishvili T, Link-Gelles R, Reingold A, Gershman K, Petit S, Farley MM, Harrison LH, Lynfield R, Bennett NM, et al. Use of surveillance data to estimate the effectiveness of the 7-valent conjugate pneumococcal vaccine in children less than 5 years of age over a 9 year period. Vaccine. 2012; 30(27):4067-72.

24. Mooney JD, Weir A, McMenamin J, Ritchie LD, Macfarlane TV, Simpson CR, Ahmed S, Robertson C, Clarke SC. The impact and effectiveness of pneumococcal vaccination in Scotland for those aged 65 and over during winter 2003/2004. BMC Infect Dis. 2008;8:53.

25. Broome CV, Facklam RR, Fraser DW. Pneumococcal disease after pneumococcal vaccination: an alternative method to estimate the efficacy of pneumococcal vaccine. N Engl J Med. 1980;303(10):549-52.

26. Menzies RI, Jayasinghe SH, Krause VL, Chiu CK, Mclntyre PB. Impact of pneumococcal polysaccharide vaccine in people aged 65 years or older. Med J Aust. 2014;200(2):112-5.

27. Andrews RM, Counahan ML, Hogg GG, Mclntyre PB. Effectiveness of a publicly funded pneumococcal vaccination program against invasive pneumococcal disease among the elderly in Victoria, Australia. Vaccine. 2004;23(2):132-8.

28. Andrews NJ, Waight PA, George RC, Slack MP, Miller E. Impact and effectiveness of 23-valent pneumococcal polysaccharide vaccine against invasive pneumococcal disease in the elderly in England and Wales. Vaccine. 2012;30(48):6802-8.

29. Yildirim I, Shea KM, Pelton SI. Pneumococcal disease in the era of pneumococcal conjugate vaccine. Infect Dis Clin N Am. 2015;29(4):679-97.

30. Chen RT, Orenstein WA. Epidemiologic methods in immunization programs Epidemiol Rev. 1996;18(2):99-117.

31. Cohen AL, Taylor T Jr, Farley MM, Schaffner W, Lesher $L$, Gershman KA, Bennett NM, Reingold A, Thomas A, Baumbach J, et al. An assessment of the screening method to evaluate vaccine effectiveness: the case of 7-valent pneumococcal conjugate vaccine in the United States. PLoS One. 2012;7(8):e41785.

32. Wang LY, Lee WC. Studying the causes of adverse drug reactions: circumvent the warning-interfered bias using the case-case comparison approach. J Clin Epidemiol. 2008;61(5):516.

33. Andrews N, Waight PA, Borrow R, Ladhani S, George RC, Slack MP, Miller E. Using the indirect cohort design to estimate the effectiveness of the seven valent pneumococcal conjugate vaccine in England and Wales. PLoS One. 2011;6(12):e28435

34. Dinleyici EC, Yargic ZA. Pneumococcal conjugated vaccines: impact of PCV7 and new achievements in the postvaccine era. Expert Rev Vaccin. 2008; 7(9):1367-94.

35. Moore MR, Link-Gelles R, Schaffner W, Lynfield R, Lexau C, Bennett NM, Petit S, Zansky SM, Harrison LH, Reingold A, et al. Effect of use of 13-valent pneumococcal conjugate vaccine in children on invasive pneumococcal disease in children and adults in the USA: analysis of multisite, populationbased surveillance. Lancet Infect Dis. 2015;15(3):301-9.

36. Weinberger DM, Malley R, Lipsitch M. Serotype replacement in disease after pneumococcal vaccination. Lancet. 2011;378(9807):1962-73.

37. Naucler P, Galanis I, Morfeldt E, Darenberg J, Ortqvist A, Henriques-Normark B. Comparison of the impact of pneumococcal conjugate vaccine 10 or pneumococcal conjugate vaccine 13 on invasive pneumococcal disease in equivalent populations. Clin Infect Dis. 2017;65(11):1780-9.

38. Balsells E, Guillot L, Nair H, Kyaw MH. Serotype distribution of Streptococcus pneumoniae causing invasive disease in children in the post-PCV era: a systematic review and meta-analysis. PLoS One. 2017;12(5):e0177113.

39. Halloran ME, Longini IM, Struchiner CJ. Design and analysis of vaccine studies. New York: Springer; 2010. p. 143-9.

40. Nemes S, Jonasson JM, Genell A, Steineck G. Bias in odds ratios by logistic regression modelling and sample size. BMC Med Res Methodol. 2009;9:56.

41. Ochoa-Gondar O, Vila-Corcoles A, Rodriguez-Blanco T, Gomez-Bertomeu F, Figuerola-Massana E, Raga-Luria X, Hospital-Guardiola I. Effectiveness of the 23-valent pneumococcal polysaccharide vaccine against communityacquired pneumonia in the general population aged $>/=60$ years: 3 years of follow-up in the CAPAMIS study. Clin Infect Dis. 2014;58(7):909-17.

42. Klugman KP. A tale of 2 pneumococcal vaccines. Clin Infect Dis. 2014:58(7): 925-7. 
43. Grabenstein JD, Manoff SB. Pneumococcal polysaccharide 23-valent vaccine: long-term persistence of circulating antibody and immunogenicity and safety after revaccination in adults. Vaccine. 2012;30(30):4435-44.

44. Musher DM, Manof SB, Liss C, McFetridge RD, Marchese RD, Bushnell B, Alvarez F, Painter C, Blum MD, Silber JL. Safety and antibody response, including antibody persistence for 5 years, after primary vaccination or revaccination with pneumococcal polysaccharide vaccine in middle-aged and older adults. J Infect Dis. 2010;201(4):516-24.

45. Pilishvili T, Bennett NM. Pneumococcal disease prevention among adults: strategies for the use of pneumococcal vaccines. Vaccine. 2015;33(Suppl 4): D60-5.

46. Centers for Disease C, Prevention, Advisory Committee on Immunization P. Updated recommendations for prevention of invasive pneumococcal disease among adults using the 23-valent pneumococcal polysaccharide vaccine (PPSV23). MMWR Morb Mortal Wkly Rep. 2010;59(34):1102-6.

47. Trucchi C, Paganino C, Ansaldi F. Methodological criticisms in the evaluation of pneumococcal conjugate vaccine effectiveness. J Prev Med Hyg. 2015; 56(3):E144-9.

48. Evers SM, Ament AJ, Colombo GL, Konradsen HB, Reinert RR, Sauerland D, Wittrup-Jensen K, Loiseau C, Fedson DS. Cost-effectiveness of pneumococcal vaccination for prevention of invasive pneumococcal disease in the elderly: an update for 10 Western European countries. Eur J Clin Microbiol Infect Dis. 2007;26(8):531-40.

49. Chang YC, Chou YJ, Liu JY, Yeh TF, Huang N. Additive benefits of pneumococcal and influenza vaccines among elderly persons aged 75 years or older in Taiwan--a representative population-based comparative study. J Infect. 2012;65(3):231-8.

\section{Publisher's Note}

Springer Nature remains neutral with regard to jurisdictional claims in published maps and institutional affiliations.

Ready to submit your research? Choose BMC and benefit from:

- fast, convenient online submission

- thorough peer review by experienced researchers in your field

- rapid publication on acceptance

- support for research data, including large and complex data types

- gold Open Access which fosters wider collaboration and increased citations

- maximum visibility for your research: over $100 \mathrm{M}$ website views per year

At $\mathrm{BMC}$, research is always in progress.

Learn more biomedcentral.com/submissions 